\title{
Le lymphocyte est une cellule endocrine
}

Les lymphocytes peuvent, après activation, synthétiser un grand nombre de récepteurs d'hormones et de neuromédiateurs qui modulent leur prolifération et leur fonction; ils peuvent aussi produire eux-mêmes certaines de ces hormones qui pourront agir sur le système nerveux central ou sur des cibles périphériques.

\section{Élisabeth Modigliani}

Professeur d'endocrinologie à la Faculté de Médecine de Bobigny (Paris XIII).

\section{Liliane Gattegno}

Chef de travaux-Praticien hospitalier de Biologie Cellulaire à la Faculté de Médecine de Bobigny (Paris XIII).

\section{RÉFÉRENCES}

1. Krug V, Krug F, Cuatrecasas P. Emergence of insulin receptors on human lymphocytes during in vitro transformation. Proc Nall Acad Sci USA 1972 ; 69 : 2604-10.

2. Payan DG, Goetzl EJ. Modulation of lymphocyte function by sensori neuropeptides. $J$ Immunol 1985 ; 135 : 782-4s.

3. Bhatena SJ, Louie J, Schechter GP, Redman RS, Wahl L, Mecant P. Identification of human mononuclear leukocytes bearing receptors for somatostatin and glucagon. Diabetes $1983 ; 30: 127-31$

4. Harel-Bellan A, Fradelizi D. Autoimmunité et interleukines. médecine/sciences $1985 ; 1$ : 313-8.

\section{ADRESSE}

E. Modigliani, L. Gattegno : service de médecine interne-endocrinologie et biologie cellulaire, hôpital Avicenne, 125, route de Stalingrad, 93009 Bobigny.

$\mathrm{m} / \mathrm{s} n^{\circ} 5$ vol. 3 , mai 87

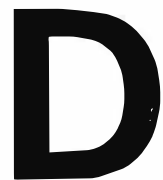
es travaux récents ont permis de démontrer l'existence d'interrelations entre les systèmes immunitaires, endocriniens et neuroendocriniens [1-3]. Non seulement le système immunitaire peut, dans certaines conditions, sécréter des interleukines et des lymphokines (interleukines 1 et 2, interféron gamma...) qui ont pour effet de moduler son fonctionnement [4] mais il possède des récepteurs spécifiques d'hormones et de neuropeptides [1-3], et il est également capable de sécréter ces composés $[5,6]$.

\section{Récepteurs lymphocytaires}

Le lymphocyte au repos ne possède pas de récepteurs spécifiques de la plupart des hormones. Au contraire, les lymphocytes $\mathrm{T}$ ou B activés possèdent à leur surface des récepteurs spécifiques de nombreuses hormones et de nombreux neuropeptides :

(a) Les premiers récepteurs individualisés ont été ceux de l'insuline [1] et de l'hormone de croissance [7]. Or, ces deux hormones ont un rôle sur la croissance des lymphocytes [7]. Les effets de l'insuline ont été particulièrement étudiés. La présence de cette hormone provoque une augmentation de la sensibilité de lymphocytes $T$ activés à l'action de la concana- valine $\mathrm{A}$ ou à celle de l'interleukine 2 [8]. L'insuline est également douée de la capacité d'accentuer la croissance de lymphocytes $\mathrm{T}$ préalablement stimulés par un antigène. L'action de cette hormone s'exerce probablement par l'intermédiaire d'un effet sur le métabolisme des lymphocytes $\mathrm{T}$ activés. En effet, en présence d'insuline, des lymphocytes $T$ activés par des mitogènes, présentent une augmentation supplémentaire de leur métabolisme [9]. De plus, une régulation négative existe in vivo entre la concentration d'insuline et la synthèse de son récepteur lymphocytaire [10]. Très récemment, on a démontré l'existence de complexes multimoléculaires entre les récepteurs lymphocytaires B humains spécifiques de l'insuline et les glycoprotéines HLA I et II. Ces complexes pourraient avoir un rôle sur la transmission des signaux hormonaux [11].

(b) Outre l'existence de récepteurs insuliniques, certaines souspopulations de cellules $\mathrm{B}$ ou $\mathrm{T}$, dans certaines conditions de différenciation, expriment des récepteurs spécifiques des facteurs de croissance ou somatomédines ou IGF.

Ces facteurs de croissance insulinlike (IGF) constituent un groupe de peptides circulants caractérisés 


\section{RÉFÉRENCES}

5. Smith EM, Menamin DH, Blalock JE. Lymphocyte production of endorphins and endorphin-mediated immunoregulatory activity. J Immunol 1985 ; 135 : 779-81s.

6. Smith EM, Blalok JE. Human lymphocyte production of ACTH and endorphin-like substances : association with leucocyte interferon Proc Natl Acad Sci USA 1981; 78 : 7530-4.

7. Snow EC. Insulin and growth hormone function as minor growth factors that potentiate lymphocyte activation. J Immunol 1985 ; $135: 776-8 \mathrm{~s}$

8. Snow EC, Feldbush TL, Oaks A. The role of insulin in the response of murine $T$ lymphocytes to mitogenic stimulation in vitro. $J$ Immunol 1980 ; 124 : 739-45.

9. Helderman JH. Role of insulin in the intermediary metabolism of the activated thymic derived lymphocyte. $J$ Clin Invest $1981 ; 67$ : 1636-45.

10. Helderman JH. Acute regulation of human lymphocyte insulin receptors. $J$ Clin Invest $1984 ; 74: 1428-35$

11. Samson M, Cousin JL, Fehlmann M. Cross-linking of insulin receptors to MHC antigens in human B lymphocytes : evidence for selective molecular interactions. $J$ Immunol 1986 ; 137 : 2293-8.

12. Lee PDK, Rosenfeld RG, Hintz RL, Smith SD. Characterization of insulin, insulinlike growth factors I and II and growth hormone receptors on human leukemic lymphoblaste. J Clin Endocrinol Metab 1986 ; 62 28-35.

13. Brown TJ, Ercolami L, Ginsberg DM. Demonstration of receptors for insulin-like growth factor II on human T lymphocytes. $J$ Recept Re.s 1985 ; 5 : 297-309.

14. Johnson HM, Smith EM, Torres BA, Blalock JE. Neuroendocrine hormone regulation of in vitro antibody production. Proc Natl Acad Sci USA 1982 ; 79 : 4226-30.

15. Payan JG, Brewster DM, Goetzl EJ. Stereospecific receptors for substance $P$ on cultured human IM-9 lymphoblasts. $J$ Immunol $1984 ; 133$ : 3260-5.

16. O'Dorisio MS, Wood CL, O'Dorisio TM Vasoactive intestinal peptide and neuropeptide modulation of the immune response. $J$ Immunol $1985 ; 135$ : 792-6s.

17. Johnson HM, Torres BA. Regulation of lymphokine production by arginine vasopressin and cytocin : modulation of lymphocyte function by neurohypophyseal hormones. $J$. Immunol 1985 ; 135 : 773-6s.

18. Meyer WJ, Smith EM, Richards GE, Cavallo A, Morrili AC, Blalock JE. In vivo immunoreactive adrenocorticotropin (ACTH) production by human mononuclear leucocytes from normal and ACTH deficient individuals.

\begin{tabular}{|c|c|c|c|}
\hline \multicolumn{4}{|c|}{$\begin{array}{c}\text { Tableau I } \\
\text { RÉCEPTEURS LYMPHOCYTAIRES HUMAINS } \\
\text { SPÉCIFIQUES DE NEUROPEPTIDES }\end{array}$} \\
\hline Réf. & Neuropeptide & $\begin{array}{l}\text { Constante de } \\
\text { dissociation }\end{array}$ & $\begin{array}{l}\text { Nombre de récep- } \\
\text { teurs par cellule }\end{array}$ \\
\hline $\begin{array}{l}{[15]} \\
{[3]} \\
{[2]}\end{array}$ & $\begin{array}{l}\text { Substance P } \\
\text { Somatostatine } \\
\text { Peptide intestinal } \\
\text { vasoactif (VIP) }\end{array}$ & $\begin{array}{l}1,8 \times 10-7 \\
0,5 \times 10-6 \\
0,47 \times 10-9\end{array}$ & $\begin{array}{r}35000 \\
250 \\
1700\end{array}$ \\
\hline
\end{tabular}

par d'étroites homologies de structure avec la pro-insuline et capables de stimuler la mitogenèse et le métabolisme cellulaire de nombreux types de cellules [12]. L'un de ces facteurs de croissance, l'IGF I, encore appelé somatomédine $\mathrm{C}$, active la synthèse d'ADN de lymphocytes humains normaux préalablement stimulés par des lectines. Des récepteurs spécifiques de l'IGF ont été mis en évidence sur des lignées de lymphocytes $\mathrm{T}$ et $B$ humains leucémiques en culture ; cependant, alors que certains lymphoblastes $\mathrm{T}$ leucémiques expriment préférentiellement les récepteurs de l'IGF I, les lymphoblastes B leucémiques étudiés [12] expriment plutôt, quant à eux, les récepteurs de l'insuline. Ceci suggère que, comme les récepteurs de l'insuline, ceux de l'IGF I pourraient être des marqueurs de différenciation lymphocytaire [12]. En ce qui concerne l'IGF II, sa liaison aux lymphoblastes leucémiques est très faible [12]. Cependant, des récepteurs spécifiques de l'IFG II sont présents sur des lymphocytes $T$ humains activés par des mitogènes comme la phytohémagglutinine, mais non sur les lymphocytes $T$ au repos [13].

(c) Actuellement, l'existence d'autres récepteurs lymphocytaires a été démontrée, comme ceux spécifiques de l'ACTH* [14], des endorphines* [5], du VIP* (peptide intestinal vasoactif) [2], de la

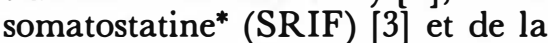
substance $P^{*}[15]$; leurs constantes d'affinité et leur nombre de sites ont été déterminés, comme le rapporte le Tableau I pour les neuropeptides [2]. Ces neuropeptides, par interaction stéréospécifique avec leurs récepteurs, agissent à des concentrations physiologiques sur le fonctionnement lymphocytaire, en activant ou en inhibant leurs transformations lymphoblastiques (Tableau II) $[2,3,16]$.

Ainsi, à extrêmement faibles concentrations $\left(10^{-10} \mathrm{M}\right.$, ce qui représente une concentration physiologique), la substance $\mathrm{P}$ stimule la transformation lymphoblastique de lymphocytes $T$ humains [15] tandis que la somatostatine a au contraire une action inhibitrice [16]. Signalons que l'ocytocine et la vasopressine sont capables d'induire la production d'interféron par des lymphocytes de souris et ce probablement par l'intermédiaire d'interactions spécifiques avec des récepteurs lymphocytaires [17].

\section{Le lymphocyte peut produire des hormones}

(a) L'ACTH et les endorphines ont été isolées de lymphocytes humains activés in vitro et de cellules spléniques de la souris in vivo. Leurs caractéristiques biochimiques, biologiques et immunologiques sont les mêmes que celles des peptides hypophysaires que nous connaissons; la naloxone bloque l'effet des endorphines pro-

\footnotetext{
- Voir glossaire p. 281.
} 


\begin{tabular}{|c|c|c|c|}
\hline \multicolumn{4}{|c|}{$\begin{array}{c}\text { Tableau } \| \\
\text { ETS DES NEUROPEPTIDES SUR L'IMMUNITÉ }\end{array}$} \\
\hline Réf. & Neuropeptides & Action globale & Expériences \\
\hline [17] & $\begin{array}{l}\text { Arginine-vasopressine } \\
\text { (AVP) ocytocine }\end{array}$ & Stimulateur & Favorise l'induction de l'interféron $\gamma$ (souris) \\
\hline$[15]$ & Substance $P^{*}$ & Stimulateur & $\begin{array}{l}\text { Rôle dans l'inflammation neurogène } \\
\text { Induit la prolifération lymphocytaire } \\
\text { Stimule le chimiotactisme } \\
\text { Augmente la prise de thymidine et de leucine par les } \\
\text { lymphocytes en culture }\end{array}$ \\
\hline$[2]$ & Neurotensine* & Stimulateur & $\begin{array}{l}\text { Vasodilatatrice } \\
\text { Libère I'histamine des mastocytes }\end{array}$ \\
\hline$[16]$ & Somatostatine (SRIF) * & Atténuateur & $\begin{array}{l}\text { Bloque l'action de la substance P } \\
\text { Diminue la prolifération lymphoblastique } \\
\text { Diminue la prise de thymidine et de leucine par les } \\
\text { lymphocytes en culture }\end{array}$ \\
\hline [16] & $\begin{array}{l}\text { Peptide intestinal } \\
\text { vasoactif (VIP)* }\end{array}$ & Atténuateur & $\begin{array}{l}\text { Active les lymphocytes suppresseurs } \\
\text { Diminue la prolifération lymphoblastique } \\
\text { Module la migration du lymphocyte }\end{array}$ \\
\hline [24] & Noradrénaline & Inhibiteur & $\begin{array}{l}\text { L'arrêt de l'innervation sympathique entraîne l'augmen- } \\
\text { tation de réponse immune }\end{array}$ \\
\hline
\end{tabular}

* Voir glossaire p. 281.

duites. En outre, de plus gros peptides tels que la proopiomélanocortine ont pu être observés dans le lymphocyte [6].

Le premier moyen de stimulation de cette sécrétion hormonale du lymphocyte est réalisé par des agents immuno-stimulants. Ainsi, l'infection de la souris par le virus de Newcastle entraîne une production splénique de bêtaendorphine [18]. L'administration de lipopolysaccharides bactériens (LPS) entraîne la production d'ACTH 1-24 et d'alpha ou de gamma-endorphine. Ces données laissent entrevoir des possibilités de régulations multiples, dans la mesure où le type de stimulus semble capable d'orienter la production hormonale à partir de la proopiomélanocortine* dans une direction déterminée $[5,6]$.

Le deuxième moyen de stimulation de la production de ces hormones par le lymphocyte sont les neuropeptides hypothalamiques : le $\mathrm{CRF}^{*}$ entraîne chez l'animal une synthèse de novo et une libération d'ACTH comme de $m / s n^{\circ} 5$ vol. 3 , mai 87 bêta-endorphine. Plus récemment, Meyer a pu mettre en évidence une augmentation du pourcentage des lymphocytes positifs pour l'ACTH chez des enfants atteints de retard de croissance et soumis à une hypoglycémie insulinique [18]. Cette régulation par l'hypothalamus est encore authentifiée par le fait que la déxaméthasone est capable de bloquer la sécrétion d'ACTH et de bêta-endorphine d'origine lymphocytaire [6]. Les implications de telles données dans la compréhension du mécanisme du stress sont évidemment très importantes.

(b) Les lymphocytes humains sont capables de sécréter un facteur susceptible d'induire la libération de la corticostérone du rat : ce facteur appelé GIF ou glucocorticoid increasing-factor n'est pas encore purifié, mais differe des lymphokines connues comme l'interféron et les interleukines. Son action surrénalienne n'est pas directe puisqu'il augmente l'ACTH [19]. Il agit donc par l'intermédiaire de l'hypophyse sur la concentration sanguine des glucocorticoïdes [20]. En effet, l'hypophysectomie ou l'administration préalable de déxaméthasone prévient les effets du GIF sur le taux sanguin des glucocorticoïdes [20-22].

(c) De plus, le lymphocyte $\mathrm{T}$, dans certaines conditions de stimulation, en l'occurence par l'entérotoxine A staphylococcique (EAS), sécrète de la TSH*. Or, la TSH stimule la réponse anticorps précoce et se comporte comme un facteur de croissance et/ou de dédifférenciation propre au lymphocyte B. Par contre, la stimulation de lymphocytes par des lipopolysaccharides n'est pas suivie de sécrétion de TSH [23]. (d) Enfin, la production de VIP et de somatostatine lymphocytaires a été plus récemment mise en évidence [16]. D'autres peptides hypophysaires peuvent potentiellement être produits par le lymphocyte, et d'autres seront

* Voir glossaire p. 281. 


\section{RÉFÉRENCES}

19. Smith EH, Meyer WJ, Blalock JE. Virusinduced increases in corticosterone in hypophysectomized mice : a possible lymphoid-adrenal axis. Science 1982 ; 218 : 1311-3.

20. Besedovsky HO, Del Rey A, Sorkin E, Lotz W, Schwulera E. Lymphoid cells produce an immunoregulatory glucorticoid increasing factor (GIF) acting through the pituitary gland. Clin Exp Immunol 1985 ; 59 : 662

21. Besedovsky HO, Sorkin E, Keller M, Müller J. Changes in blood hormone levels during the immune response. Proc Soc Exp Biol Med 1975 ; 150 : 466.

22. Besedovsky HO, Del Rey A, Sorkin E. Antigenic competition between horse and sheep red blood cells as a hormone-dependent phenomenon. Clin Exp Immunol 1979 ; 37 : 106.

23. Smith EM, Phan M, Kruger TE, Cooppenhaver DH, Blalock JE. Human lymphocyte production of immune reactive thyrotropin. Proc Natl Acad Sci USA 1983 ; 80 : 6010-3.

24. Besedovsky HO, Adriana EM, Sorkin E. Immune-neuroendocrine interactions. $J$ Immunol $1985 ; 135: 750-4 \mathrm{~s}$

25. Smith EM, Johnson HM, Blalock JE. Lymphocytes as a peripheral source of and target for endogenous opiates. In : Fraiolo F, Isidori A, Mazzetti M, eds. Opioid Peptides in the Periphery. Amsterdam : Elsevier Editors, 1984 : 129.

26. Blalock JE, Harbour-McMenamin D, Smith EM. Peptide hormones shared by the neuroendocrine and immunologic systems. $J$ Immunol $1985 ; 135: 858-61 \mathrm{~s}$.

27. Dupont AG, Somers G, Van Steirleghen AC, Warson F, Vanhelot L. Ectopic adrenocoticotropin production : disappearance after removal of inflammatory tissue. $J$ Clin Endocrinol Metab 1984 ; 58 : 654-8.

28. Hall NR, Gillis JMC, Spangelo BL, Goldstein AL. Evidence that thymosins and other biologic response modifiers can function as neuroactive transmitters. $J$ Immunol 1985 ; $135: 806-10$ s.

29. Dianrello CA. Interleukin 1 and the pathogenesis of the acute phase response. $N$ Engl J Med 1984 ; 311 : 1413-8.

30. Blalock JE, Smith EM. Human leukocyte interferon (HU I Fn): potent endorphin-like opioid. Biochem Biophys Res Commun 1981 ; 101 : 472-6.

31. Wolosky BM, Smith RNJ, Smith EM, Meyer WJ, Fuller GM, Blalock JE. Corticotropin releasing activity of monokines. Science $1985 ; 230$ : 1035-7.

32. Shavit Y, Terman QW, Martin FC, Lewis JW, Liebeskind JC, Gale RP. Stress, opioid peptides, the immune system and can- peut-être isolés dans un proche avenir [24-26].

Cette production directe d'hormones par le lymphocyte permet un schéma de régulation double avec la présence d'un axe neuro-hypothalamo-immuno-hypophysaire dont l'existence est prouvée pour l'axe corticotrope (figure 1).

La production d'ACTH par le lymphocyte sous l'influence de stimuli non cognitifs est capable de freiner la production hypophysaire d'ACTH. Inversement, des stimuli cognitifs, émotionnels en particulier, peuvent faire sécréter du CRF qui stimule l'ACTH et les endorphines lymphocytaires $[24,25]$. L'importance quantitative respective de ces deux axes reste à préciser. Néanmoins, une confirmation en pathologie a pu être déjà apportée par Dupont en 1984 ; cet auteur a en effet décrit chez l'homme un syndrome de

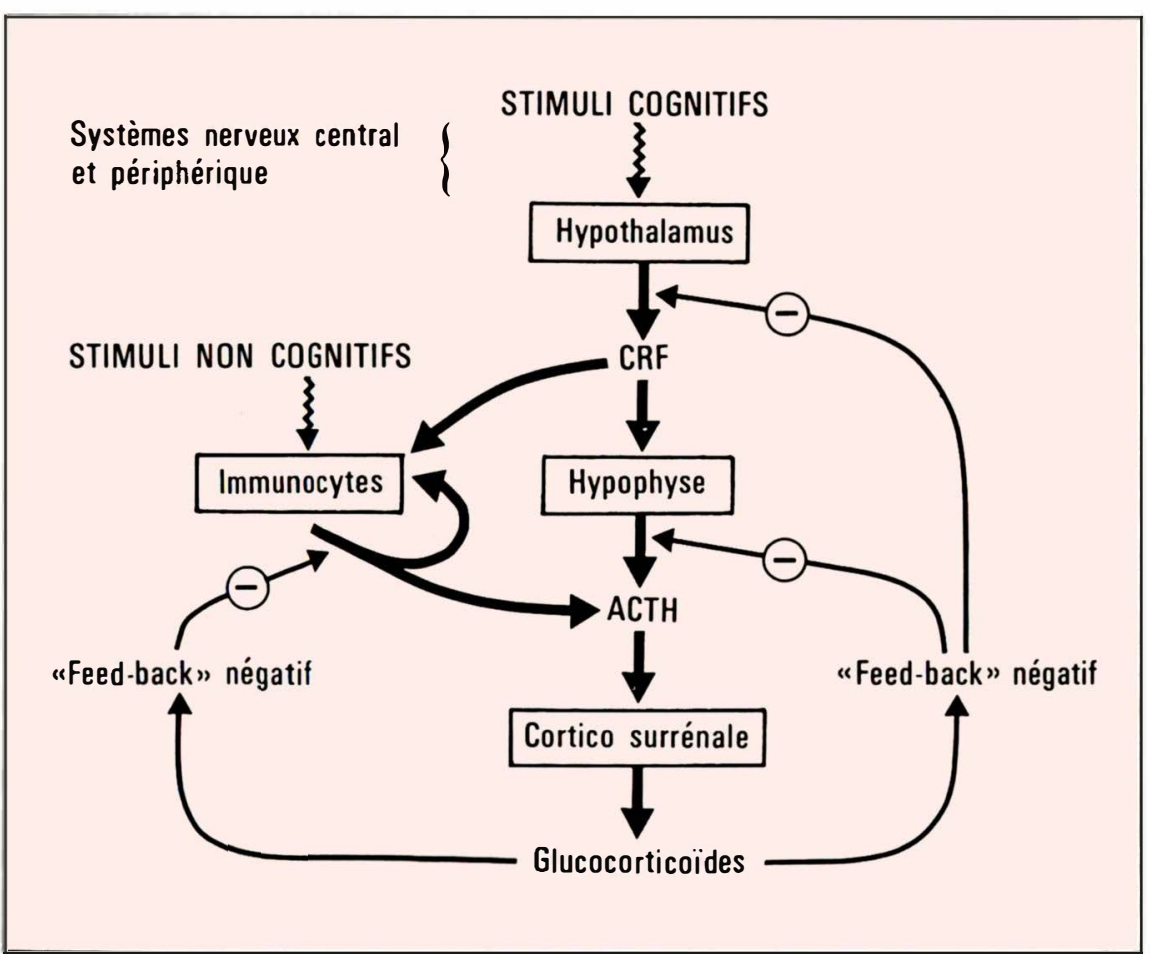

Figure 1. Axe immuno-hypothalamo-hypophysaire (D'après [25]). Des stimuli cognitifs (stress) provoquent la sécrétion par l'hypothalamus de CRF (corticotropin releasing factor) agissant sur l'hypophyse et sur les immunocytes et induisant la sécrétion d'ACTH (adrenocorticotropin hormone) à la fois par l'hypophyse et par les lymphocytes. Des stimuli non cognitifs (immunostimulants) sont également capables de provoquer la sécrétion d'ACTH lymphocytaire. Les glucocorticoïdes sécrétés exercent alors des mécanismes de rétrocontrôle (feed-back) négatifs. production ectopique d'ACTH qui était secondaire à une lésion inflammatoire (lipogranulome paravertébral) et qui a rétrocédé à l'ablation de cette tumeur [27]. La même double régulation existe pour les endorphines (figure 2) avec production de lymphokines et effet propre des endorphines à différents niveaux [5].

\section{Rôle de ces hormones sur l'immunité}

Il est logique de proposer une finalité à cette production hormonale. De fait, l'action inhibitrice des glucocorticoïdes sur la réponse anticorps est connue de longue date, plus marquée sur les cellules au repos. Ces glucocorticoïdes sont capables de s'opposer aux phénomènes de compétition antigénique $[21,22]$. On conçoit ainsi leur rôle dans la limitation de l'activation du lymphocyte. Mais l'ACTH a aussi une action pro- 
pre d'immunodépression retrouvée chez le rat surrénalectomisé. Elle a une action inhibitrice sur la production d'anticorps après stimulation antigénique in vivo et in vitro. Elle inhibe la mitogenèse et freine la production d'interféron gamma induite par l'entérotoxine staphylococcique A dans la rate de souris. Johnson a de plus isolé un composé voisin de l'ACTH, qui a une action inhibitrice encore plus marquée [14].

Les effets des endorphines sont plus divergents [5] et les résultats parfois contradictoires (Tableau III). La bêta-endorphine n'a en règle générale pas d'action immunosuppressive, à l'opposé de l'alpha-endorphine [5]. Or, selon le stimulus immunogénique, la proopiomélanocortine peut être scindée de différentes manières, ce qui fait entrevoir une possibilité de modulation très fine de la réponse immune.

Une telle intrication entre système nerveux endocrinien et immun relève vraisemblablement d'un schéma très général. En effet, beaucoup de neuropeptides situés dans le système nerveux périphérique, pour lesquels les lymphocytes possèdent des récepteurs de façon certaine, et qu'il produit probablement lui-même, ont une action de modulation des fonctions immunes locales [16]. C'est ce que rappelle très schématiquement le Tableau II.

Inversement les produits sécrétés par le système immunitaire et/ou ayant une action sur le système immunitaire s'avèrent avoir une action sur le système neuroendocrinien.

C'est ainsi qu'on a pu mettre en évidence [28] : (a) une modification des phases du sommeil sous l'effet de l'interleukine 1 qui agit également sur les centres thermiques et le comportement et diminue le contenu hypothalamique en norépinéphrine [29]; (b) des modifications telles que léthargie et dépression induites par l'interféron [30] ; (c) et surtout, une augmentation de l'ACTH hypophysaire sous l'effet de l'interleukine 1 [31] ; cette action $\mathrm{m} / \mathrm{s} \mathrm{n}^{\circ} 5$ vol. 3, mai 87

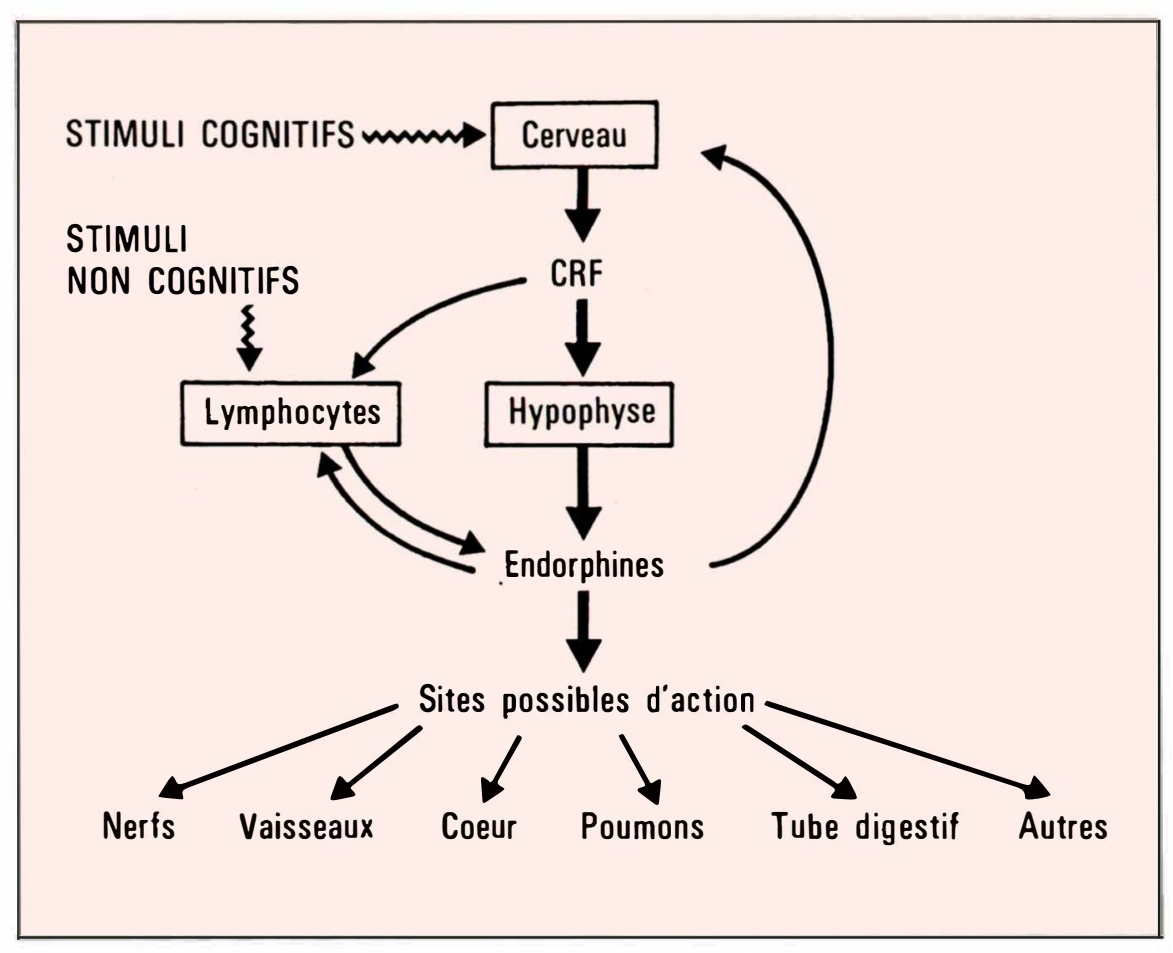

Figure 2. Inter-relations entre le système immunitaire et le système nerveux central par I'intermédiaire des endorphines ( $D$ 'après [5]). Des stimuli cognitifs (stress) provoquent la sécrétion par l'hypothalamus de CRF (corticotropin releasing factor) agissant sur l'hypophyse et sur les immunocytes et induisant la sécrétion d'endorphines à la fois par l'hypophyse et par les lymphocytes. Des stimuli non cognitifs (immunostimulants) sont également capables de provoquer la sécrétion d'endorphines lymphocytaires. Les endorphines ainsi formées agissent sur les lymphocytes (qui possèdent des récepteurs spécifiques) et sur d'autres cellules.

Tableau III

ACTION DE L'ACTH ET DES ENDORPHINES SUR LE SYSTĖME IMMUNITAIRE

\begin{tabular}{|c|l|c|c|c|c|c|}
\hline Réf. & & ACTH* & $\begin{array}{c}\beta \text {-Endor- } \\
\text { phine }\end{array}$ & $\begin{array}{c}\gamma \text {-Endor- } \\
\text { phine }\end{array}$ & $\begin{array}{c}\alpha \text {-Endor- } \\
\text { phine }\end{array}$ & $\begin{array}{c}\text { Enképha- } \\
\text { line (MET) }\end{array}$ \\
\hline$[5]$ & Activité NK** & & $\uparrow$ & $\uparrow$ & 0 & $\uparrow$ ou 0 \\
\hline$[14]$ & $\begin{array}{l}\text { Synthèse d'anti- } \\
\text { corps (plaquefor- } \\
\text { ming cell) }\end{array}$ & $\downarrow$ & 0 & \pm & $\downarrow 50 \%$ & $\downarrow$ \\
\hline$[5]$ & Prolifération & $\downarrow$ & $\uparrow$ ou $\downarrow$ & & 0 & $\uparrow$ \\
\hline$[14]$ & $\begin{array}{l}\text { Production d'in- } \\
\text { terféron }\end{array}$ & $\downarrow 60 \%$ & & & & \\
\hline
\end{tabular}

* Action indirecte par production de glucocorticoïdes, mais aussi action directe.

* * Voir glossaire p. 281. 
est aussi puissante que celle du CRF ou de l'arginine vasopressine [31] et paraît voisine de celle du GIF.

Plus précise encore est l'action neurohormonale des thymosines* [28] sécrétées par les cellules épithéliales thymiques et qui sont par ailleurs connues pour leur stimulation des lymphokines*. La thymosine a un rôle de stimulation in vitro de l'axe hypothalamohypophyso-surrénal (thymosine alpha 1 + F5). Elle a une action opposée à celle des glucocorticoïdes : elle augmente la réponse à la concanavaline A de lymphocytes spléniques traités par l'hydrocortisone. Elle agirait soit en inhibant le CRF, soit en interférant avec le rétro-contrôle négatif des glucocorticoïdes sur ce CRF. En effet, elle inhibe la liaison de la déxaméthasone marquée sur les lymphocytes [28]. La thymosine permet une transformation des lymphocytes immatures en lymphocytes matures, moins sensibles à l'action des glucocorticoïdes, ce qui lui permet de jouer un rôle de modulateur.

La régulation du stress est désormais bien différente de celle admise il $y$ a quelques années.

Il était admis que le stress stimulait l'axe CRF-ACTH-cortisol et que la production de glucocorticoïdes induite avait une action

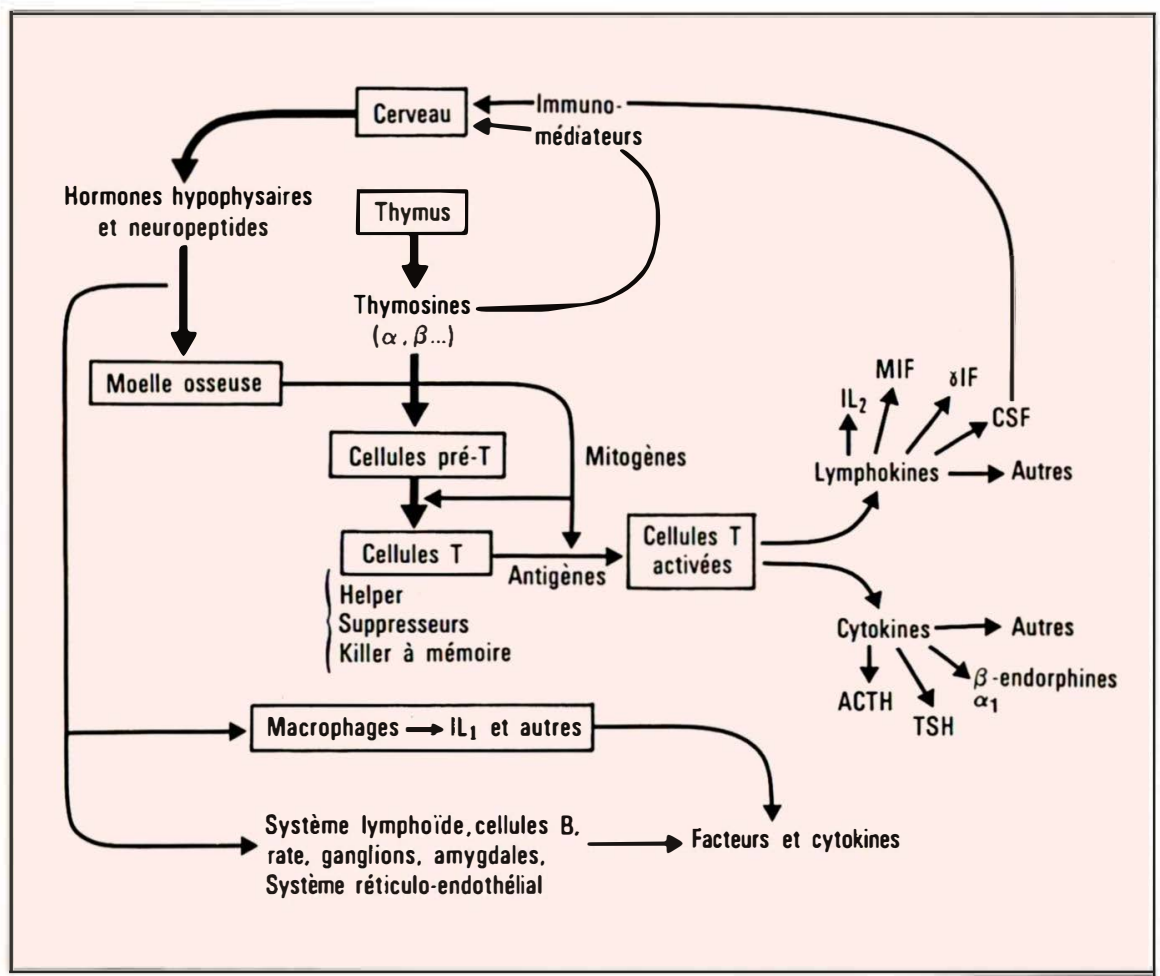

Figure 3. Inter-relations possibles entre systèmes immunitaire et neuroendocrinien ( $D^{\prime}$ après [27]). Le cerveau est un lieu de sécrétion d'hormones hypophysaires et de neuropeptides agissant sur les lymphocytes par l'intermédiaire de récepteurs. Les thymosines, hormones thymiques, sont nécessaires a la maturation des cellules $T$. Ces cellules $T$, sous l'effet de mitogènes ou $d^{\prime}$ antigènes sécrètent non seulement des lymphokines.comme I'IL $L_{2}$ (inter-

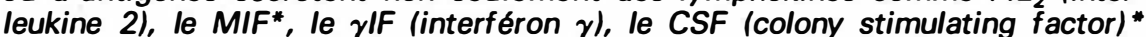
mais également des cytokines comme I'ACTH (adrenocorticotropin hormone), la TSH (thyreostimulating hormone) ou les $\alpha$ et $\beta$-endorphines. Les macrophages présentant I'antigène (en association avec HLA II) aux cellules $T$, sécrètent I'IL, (interleukine 1). Ce médiateur agit sur des lymphocytes et sur d'autres cellules. Les lymphocytes B sont également doués de la propriété de sécrétion de cytokines *. Lymphokines *, cytokines et médiateurs exercent en dépressive sur l'immunité. Or, il est actuellement démontré que certains stress, infectieux tout particulièrement, peuvent activer directement la production d'ACTH et d'endorphines lymphocytaires $[5,6,24]$. Les phénomènes survenant lors du stress peuvent être analysés de la façon suivante $[5$, 32] :

- production d'endorphines alpha ou gamma qui stimulent l'activité NK (natural killer)* ${ }^{*}$, donc la réponse immune ; production également de bêta-endorphine, qui, elle, est non immunosupressive mais responsable de pyrexie et d'hypotension ;

- production d'ACTH avec inhibition secondaire de la réaction immune par les glucocorticoïdes ainsi libérés ;

- sécrétion par le lymphocyte activé de lymphokines ayant leur action propre sur l'immunité.

$\mathrm{La}$ thymosine agit enfin comme modulateur supplémentaire pouvant produire la transformation du lymphocyte immature en lymphocyte mature insensible aux glucocorticoïdes. Elle peut de plus s'opposer partiellement à l'action de ces stéroïdes. Toutes ces hormones sont régulées par rétrocontrôle (feed back) : leur production freine secondairement leur libération.

\section{Conclusion}

Au total, des concentrations physiologiques d'hormones sont capables de moduler la réponse immunitaire, probablement par interaction stéréospécifique avec des récepteurs lymphocytaires. A l'inverse certaines lymphokines, hormones ou neuropeptides produits par les lymphocytes sont capables de moduler le fonctionnement du système neuroendocrinien. Ceci suggère que le lymphocyte, en réponse à des agressions diverses (infection, tumeur, agents chimiques), serait capable d'envoyer des signaux au cerveau et à d'autres organes, modifiant ainsi les réponses de l'hôte (figure 3)

\footnotetext{
- Voir glossaire, p. 281.
} 


\section{${ }^{*}$ GLOSSAIRE*}

Lymphokine : facteur soluble libéré par des lymphocytes sensibilisés, en contact avec leur antigène spécifique. MIF : lymphokine, inhibant la migration des macrophages.

Interleukine : ce terme désigne certaines lymphokines et monokines (substances sécrétées par les cellules mono-macrophagiques) agissant sur certaines populations lymphocytaires. Cependant, certaines interleukines peuvent être produites par des cellules non leucocytaires et agir non seulement sur des lymphocytes mais sur d'autres types cellulaires. Ainsi a été créé le terme de cytokine qui est plus général. Cytokine : médiateur sécrété par des cellules lymphocytaires et d'autres cellules et agissant sur des cellules lymphocytaires et d'autres cellules.

Interleukine 1 (IL $)$ : glycoprotéine sécrétée par les cellules monomacrophagiques et par d'autres cellules (kératinocytes, astrocytes...), elle agit sur les lymphocytes (en induisant la synthèse d'interleukine 2) et sur d'autres cellules.

Interleukine $2\left(\boldsymbol{I L}_{2}\right)$ : facteur de croissance des lymphocytes $T$.

CSF (Colony Stimulating Factors) : famille de facteurs permettant la prolifération et la différenciation de cellules souches hématopoḯtiques. Parmi ces facteurs, le GM-CSF (Granulocytes, Macrophage-CSF) et l'interleukine 3 sont des lymphokines.

Interféron (INF $\gamma$ ou $\gamma \boldsymbol{\gamma}$ IF) : lymphokine de structure protéique, douée d'activité antivirale, immunomodulatrice, et antitumorale et sécrétée par les lymphocytes $T$ dans certaines conditions.

Thymosine $\alpha, \beta \ldots$ : hormone de structure poly peptidique, sécrétée par les cellules épithéliales thymiques et intervenant dans la différenciation des précurseurs médullaires des cellules $T$.

Cellules $K$ ou cellules "killer": cellules mononucléees, ne possédant aucun des marqueurs des cellules $B$ ou $T$ et ayant le pouvoir d'exercer le phénomène d'ADCC ou cytotoxicité cellulaire dépendant des anticorps, c'est-à-dire la propriété de lyser des cellules cibles recouvertes de faibles quantités d'anticorps $\operatorname{Ig} G$.

Cellules NK ou cellules " natural killer" exerçant une activité $N K$ : cellules lym phoïdes possédant certains marqueurs de la lignée $T$ et capables d'exercer une action cytotoxique vis-à-vis de cellules cibles, en général tumorales, indépendamment de toute sensibilisation préalable et de toute restriction par le complexe majeur d'histocompatibilité. CRF (Corticotropin Releasing Factor) : facteur hypothalamique permettant la libération d'ACTH ainsique de POMC par l'hypophyse.

POMC (Proopiomélanocortine) : polypeptide de 241 acides aminés, précurseur commun de plusieurs peptides contenus dans sa composition moléculaire. Il est produit par le lobe antérieur de l'hy pophyse (clivé en $A C T H, \beta L P H$, bêta-endorphine...) ; mais aussi par le lobe intermédiaire et le système nerveux central (clivé en MSH, $\gamma L P H$, bêta-endorphine...).

ACTH (Adrenocorticotropin Hormone) : hormone antéhypophysaire permettant la libération du cortisol.

Bêta-endorphine : peptide opioïde de 31 acides aminés, produit dans l'antéhypophyse, mais aussi dans le lobe intermédiaire (forme acétylée) et dans le cerveau.

Metenképhaline : peptide opioide dont la formule est constituée de la partie amino-terminale de la bêta-endorphine, présente dans l'hypo physe et le cerveau, mais qui n'est pas un produit de maturation de la POMC.

TSH : hormone thyréo-stimulante d'origine hypophysaire.

VIP (peptide intestinal vasoactif) : peptide isolé dans l'intestin grêle mai sil est en fait un neurotransmetteur présent dans les neurones du système nerveux central et dans les vésicules synaptiques des terminaisons nerveuses.

SRIF (somatostatine) : peptide isolé de l'hypothalamus et mis en évidence pour son action d'inhibition sur l'hormone de croissance antéhy pophysaire ; en fait, il se retrouve dans d'autres organes (pancréas); il agit lui aussi comme un neurotransmetteur et est présent dans tous les neurones sensitifs.

Substance P et neurotensine : neurotransmetteurs de très faible poids moléculaire se trouvant également dans les terminaisons nerveuses.

Arginine vasopressine et ocytocine : neurohormones issues de la posthypophyse.

\section{Summary}

Physiological concentrations of hormones are able to modulate immune response, probably through specific interaction with lymphocyte receptors. Conversely, some lymphocyte products (lymphokines, hormones or neuropeptides) could modulate the neuro-endocrine system function. This suggests that lymphocytes might be able to send signals to the brain and to other organs, thereby modifying host responses.

\section{TIRÉS A PART}

E. Modigliani : service de médecine interneendocrinologie, hôpital Avicenne, 125, route de Stalingrad, 93009 Bobigny. 